\title{
Wearable learning tools
}

Jerry Bowskill and Nick Dyer

BT Advanced Communications Technology Centre, Adastral Park, UK, email:jerry.bowskill@btcom

In life people must learn whenever and wherever they experience something new. Until recently computing technology could not support such a notion, the constraints of size, power and cost kept computers under the classroom table, in the office or in the home. Recent advances in miniaturization have led to a growing field of research in 'wearable' computing. This paper looks at how such technologies can enhance computer-mediated communications, with a focus upon collaborative working for learning. An experimental system, MetaPark, is discussed, which explores communications, data retrieval and recording, and navigation techniques within and across real and virtual environments. In order to realize the MetaPark concept, an underlying network architecture is described that supports the required communication model between static and mobile users. This infrastructure, the MUON framework, is offered as a solution to provide a seamless service that tracks user location, interfaces to contextual awareness agents, and provides transparent network service switching.

\section{Introduction}

The world of telecommunications is being rapidly redefined by the Internet. Longer-term trends show that mobile, networked computers will form the basis of the future of personal communications. Not only is the telephone handset becoming mobile, by default, but the boundary between phone and computer is set to disappear. Networks for mobile devices are also changing fast, within the next five years the global mobile network will support megabit rather than the current kilobit connections. In summary most phones are becoming mobile, phones are becoming computers and the underlying network is becoming a fast global TCP/IP network.

Since the birth of computers, it has been a design goal of hardware manufacturers to make systems as small and compact as possible. This led on to the migration from monolithic mainframe computers to desktop workstations, and then to portable computers. In more recent years a trend has emerged for full function 'palmtop' computers, such as the Toshiba 


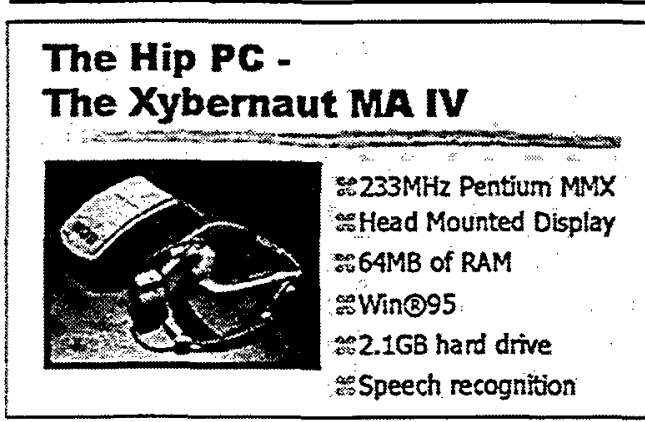

Figure 1a: Xybemaut wearable computer

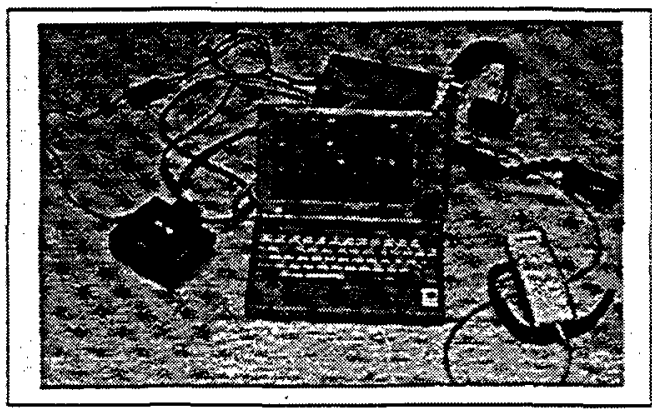

Figure Ib: A modified polmtop computer

Libretto, and even 'wearable' computers (Starner, Mann, Rhodes, Levine, Healey, Kirsch, Picard and Pentland, 1997). Today wearable computers allow us to explore the applications that will be widely accessible in the near future.

The authors use the term wearable to refer to all Information and Communication Technology (ICT) devices that are designed to be mobile. While portable computers are carried to a location and used when the user is stationary, wearable computers are designed to be used whilst the user is moving around and active in other tasks. Most wearables today are belt-mounted computers or palmtops (see Figures $1 \mathrm{a}$ and $1 \mathrm{~b}$ ). The authors believe such devices, in combination with location and other forms of 'context' awareness, enable 'ubiquitous communications' in which the user is given appropriate communications channels and multimedia information based on their location and task within a real world environment.

The implications of this for learning are significant; a 'contextual' wearable device would have the potential to deliver timely information, in an appropriate medium and at an appropriate level of detail. Another research focus is on using wearables to allow people in a real world environment to access remote people and multimedia information held within an online collaborative virtual environment. The concept of fusing real and virtual space, a form of collaborative mixed reality, is being explored through the development of an educational environment called 'MetaPark'. MetaPark is currently under construction and consists of a collaborative virtual environment modelled on a real park that is used by schools in central Ipswich as a learning resource.

Most applications for commercial wearable computers, typically belt-mounted PCs running the Windows operating system, have been restricted to tasks involving mobile data input or retrieval. However, body-worn networked computing devices are suited to many applications and the impact on personal communications will be significant. For example, a networked wearable computer can behave as a mobile phone, audio graphic conferencing terminal, a video conference terminal or as a virtual conferencing terminal (Walker and Sheppard, 1997). Let us not forget the mobile phone itself, the most widely used, networked wearable on the planet. Although the physical device has a limited multi-modal interface it can act as a 'thin client' to network embedded services, for example allowing users to send and receive email, fax and video.

Much of the current research activity around wearables is addressing 'contextual information services', primarily 'mobile location services'. These aim to deliver timely and location specific media, such as traffic reports or tourist information, to either mobile phones or palmtop PC 
terminals (Pascoe, 1998). Our research focus is on the application of wearables to computer supported collaborative working, achieving 'ubiquitous telepresence' as a result of combining traditional audio-visual conferencing with collaborative virtual environments. MetaPark is the practical embodiment of these ideas, a persistent online space that provides contextually appropriate access to colleagues, services and information. In concept this is similar to the idea of 'co-habited mixed realities' (Van de Velde, 1998); as 'wearers' move through the real world they also move through either literal or abstract virtual environments. For example, when entering our research laboratory the wearer is connected to an augmented reality conferencing session with other team members who are working remotely.

The goal is to deliver 'seamless service': a repurposing of interface and content, which delivers appropriate functionality to the user across a range of devices and in a variety of settings. The vision is of 'ubiquitous telepresence', sharing many features with the earlier work on 'ubiquitous computing' (Weiser, 1991), and 'ubiquitous media' (Buxton, 1997). Our vision of wearables as contextual communications devices is being explored based on our previous research and subjective evaluations of wearable communications. A Wearable Communication Space, WearCom (Billinghurst, Bowskill, Jessop and Morphett, 1998) for example, allows a wearable computer user to communicate with multiple remote people in a virtual environment that supports high quality audio communication, visual representations of the collaborators and an underlying spatial metaphor. Preliminary indications suggest that spatial cues significantly improve the effectiveness of multiparty conferencing, which is particularly significant for mobile communications as the user has typically more distractions than a desk-bound user. In previous research, we found that users can locate information more rapidly with this type of spatial information display than the more traditional desktop metaphor information displays (Billinghurst, Bowskill, Dyer and Morphett, 1998). Spatial displays also scored more highly during subjective evaluations. Taking an educational fieldtrip scenario, we then looked at how real and virtual environments could be connected together with wearable devices acting as the technological bridge, and this is illustrated below, in the next section.

\section{MetaPark - an overview}

MetaPark has evolved after a number of years' research into the use of collaborative virtual learning environments using a variety of interface and interaction modalities, including interactive video environments (IVE) and wearable augmented reality information spaces. In developing the MetaPark concept we wanted to demonstrate the benefits of using wearable computing technology within an educational context. The common requirements of wearables for learning are:

Information Retrieval - The ability to retrieve stored information based on current location, task or other forms of context represents the 'killer application' for mobile devices. A user should also be able to use the device to make their own queries in the same way as they would via a conventional Internet search engine (for example a text search).

Navigation - Knowing where you are (and where other people and resources are) is also a critical requirement for wearables. In addition to conventional satellite navigation, the ability to guide people was seen as desirable, particularly within a defined context such as a visit to a museum. 
Figure 2: The MetoPark world

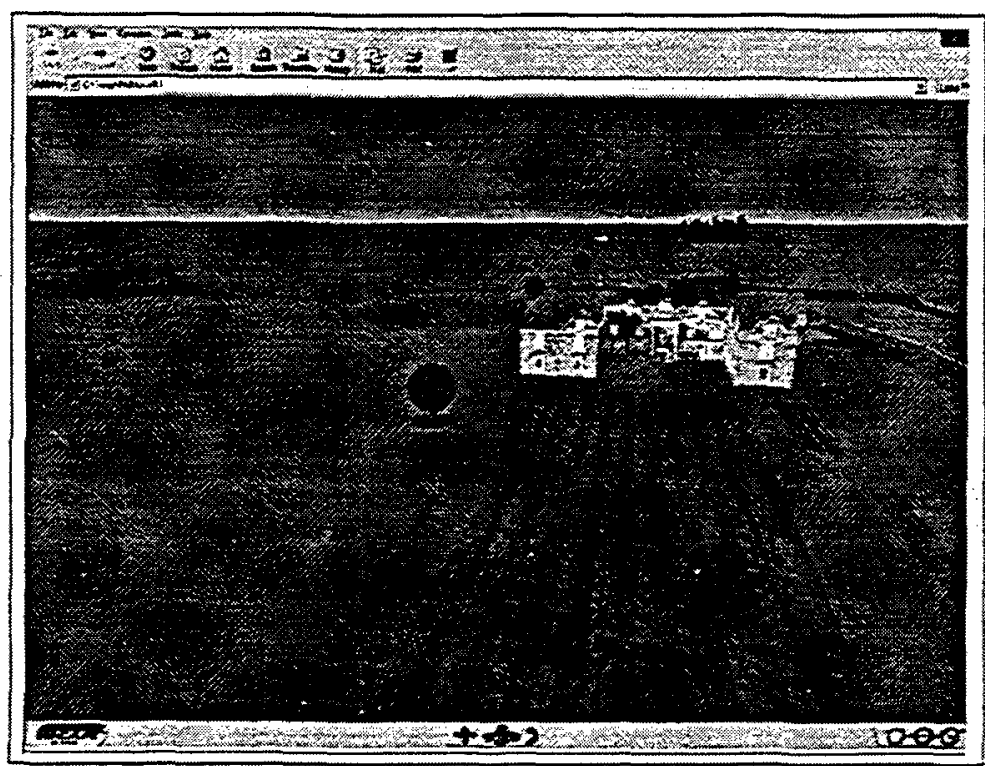

Information Capture - The ability to capture images, sounds, 'notes' and sketches. These could be stored remotely, locally and accessed on demand.

Communications - Conventional 'synchronous' audio, asynchronous 'text chat' and email messaging and the ability to send video to support communications with others. Many of the children in our focus group are members of text chat communities on the Internet.

Recreation - A learning device should be fun and rewarding to use. A large number of children in our focus group saw wearables as an extension of current hand-held gaming devices, but being used in context.

MetaPark was designed to focus upon the effectiveness of communication, information retrieval, navigation and information recording. The central idea is to create a 'cohabited mixed reality' using a literal virtual representation of a real world park which is accessible either online or by moving through the real world space itself. The virtual model of Christchurch Park, Ipswich, which forms the basis of the MetaPark concept, is shown in Figure 2.

Local schools were consulted and confirmed that they could see the potential for MetaPark, both as being a framework for continuous education and also as providing real support for fieldtrips. It is envisaged that for a MetaPark study a class of children will be split into two groups, one of which will remain in a classroom, whilst the other is taken to a location of interest (in this case Christchurch Park, Ipswich). The field workers at the park will be given wearable computers that will contain park-related information, communications software, a GPS unit and data-recording utilities. The group in the classroom will work on a computer that has the $3 D$ representation of the park as a real-time simulation. Our intention is for the children at both locations to use the same wearable interface devices although the classroom devices will be used to navigate a virtual world displayed on a large room-based display. 


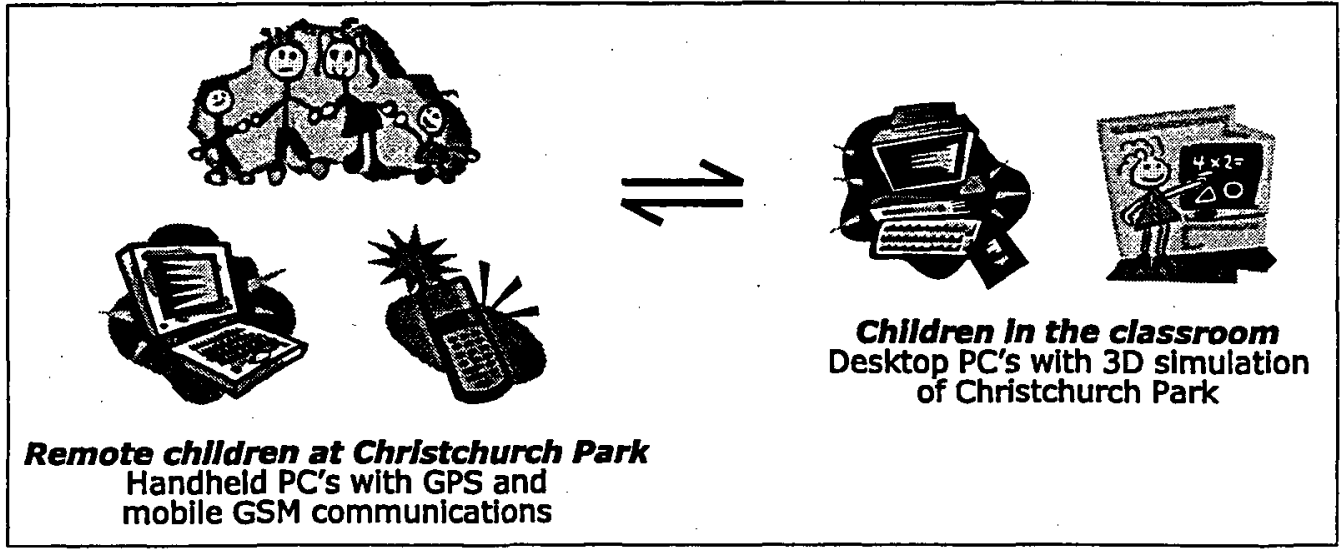

Figure 3: A MetaPark overview

The positional data from the remote GPS system will be used to place a virtual representation of the remote group within the 3D-park model. This will allow the class group to follow the path of the remote group as they explore the environment, as well as allowing the path data to be saved. The communications software will allow the remote and the class children to text chat and talk to each other so that each group can exchange information and ideas as well as taking location-specific notes (Figure 3).

In order to promote information exchange and communications between the children, the content would have to be contrived such that the remote and classroom groups receive different types of information and each group would be given different objectives which rely upon help from the opposite group.

To facilitate the technical communications between each computer unit, we are designing a network infrastructure that supports text chat and binary file transfer, and additionally allows transparent transitions between different service providers (wireless LAN, modem, infrared). Known as the MUON Framework (see below), this network architecture also tracks the user's location, based upon a predefined beacon list, and will also provide rudimentary information about the user's current level of activity. Using these cues, the classroom group should be able to alter their communications strategy as the remote group undergoes different state and location changes. The virtual environment running within the classroom is not limited to a single connection. It will also support remote collaborative access, so that other groups can enter the MetaPark - providing further input to the children's activities by communicating with the remote group as well as other virtual groups.

\section{The MUON framework}

In order to support the communications model for the MetaPark project, it was necessary to design a network architecture that allowed mobile users to communicate without the need to understand the underlying technical issues. The solution was a client/server network support system called the MUON (Mobile User Object Notification) framework. The details of the MUON framework architecture are discussed in a previous publication 
(Dyer and Bowskill, 1999); however the features that it supports, and those that underlie many future wearables applications, are outlined below.

\section{Synchronous and asynchronous communications}

The most common form of asynchronous communication is email. The MUON framework supports a similar asynchronous messaging system that allows users to send text and binary (pictures, files, etc.) data to offline recipients. This data is then stored on a server in the recipient's persistent database, ready for when they next log on. When two (or more) users are online simultaneously, a dialogue can be arranged between each client. This semisynchronous communications medium is more commonly known as text-chat. For synchronous communications, both users must be online and have enough available bandwidth to cope with the continuous stream of data between them. Audio and video communications channels are then possible. The MUON framework will not provide synchronous communications services directly, but will support external IP-based services by negotiating the connection and disconnection processes between the users.

\section{Location awareness}

To facilitate location awareness, the client requires a mechanism that determines its current position within a pre-defined mapping of physical space. This is tackled by the use of GPS for outdoor locations and radio location beacons and client receiver modules for indoor locations. The beacons are autonomous units that transmit a specific code that is used to denote location. These devices are inexpensive and can transmit a signal that can be received with sufficient accuracy even where physical obstructions cause radio attenuation. The major disadvantage is that these devices all transmit the same frequency and can therefore cause interference when used in close proximity with each other. To alleviate the problem, the strength of the beacon must be balanced between providing adequate coverage for each specific location, and enough attenuation to prevent interference with neighbouring beacons. From a software viewpoint, a primary server maintains a master database of each named location matched with its respective beacon code. When a client receives a beacon message (by passing close enough to a beacon for the receiving device to interpret a location code), it passes this up to the server, which replies with the matching location name. In this manner, the server can track each client as it moves around its environment and a user can interrogate the system to find the location of another client. The issue of privacy is managed by allowing users to switch dynamically between broadcasting or hiding their location as they see fit.

\section{Location-based messaging}

With the ability to determine location, the network can also provide another service: location-based messaging. This is where a message can be directed to a location instead of an individual user. As users with location tracking devices move around their environment, they receive messages that have been 'posted' to their current location. Conversely, they may post messages at specific locations. To illustrate, a location message could be posted to someone's desk to inform passers by (or those specifically looking for them) that they are on leave for the next two weeks, and should be contacted via email only for important messages. The location messages are sent to the server, and stored in the beacon database. When messages have expired, the server deletes them accordingly. Each message is assigned a unique ID, that is used to amend a user's database each time they read a location message, to prevent continuous notifications being sent for messages they have previously received. 


\section{Transparent network switching}

One of the key functions provided by the MUON framework is network switching. This is the ability to switch automatically between different service providers when either the connection drops, or a better bandwidth is available under a different service provider. The supported service providers are wired LAN, wireless radio LAN, infrared via serial, direct serial connection, and modem (including GSM mobile modem). Clients can select the best service providers available at log-on time, and monitor the availability of other services based on a pre-defined prioritized list.

\section{Availability and contextual awareness}

The nature of wearable computers dictates that the user can be in many states of availability according to their current situation. It is also the job of the wearable computer to determine which is the most appropriate form of feedback to notify the user of system events (such as new messages). For example it may be inappropriate for a user to receive a visual notification if they are running down a street; an audio notification would be safer! Also if the user were engaged in a meeting, it would be appropriate for the wearable computer to limit the availability of the user to receiving urgent messages only. Although these issues are not addressed directly by the MUON framework, one design is to allow MUON clients to employ the features of agent technologies that provide user availability information, and allow the system to respond to this information accordingly. Switching the context of the messages could be controlled by an external agent. The agent would feed information into the MUON client, allowing visual and audio notifications to be triggered appropriately. The provision for this type of service is accounted for by the use of 'gateways', which connect the primary server to agent servers allowing information to be exchanged between various applications. This allows clients to utilize these services without the need to have a predefined IP address (as some of the agent servers require this) or be continuously connected to the network, as the primary server (which does have a unique IP address) acts as the go-between.

\section{Discussion}

The MetaPark concept provides avenues for a number of research threads. While the MetaPark environment is still being developed, the authors have been able to demonstrate elements of the system. For example the underlying network framework has been implemented, sample collaborative virtual environments have been constructed and an intuitive mechanism for interfacing between personal devices and large displays or real world objects has been demonstrated.

From a technical viewpoint, the network architecture that supports remote communication between the real and virtual MetaPark visitors provides a technical challenge of significant complexity. When the system is functionally complete a more intensive period of evaluation will commence. In particular the authors hope to understand the relationships between the virtual and real space communities, the types of learning that occur and the communications mechanisms used. In terms of evaluation, longitudinal studies will be important in assessing the benefits of applications that sound intriguing on paper and make compelling demonstrations. Such work will provide qualitative understanding of the practical appeal of ubiquitous telepresence, but with radically new hardware there is also 
scope for more constrained, quantitative assessment of the options in presenting and interacting with wearable interfaces.

Finally, although the first instantiation of the MetaPark concept was realized for children, its underlying technologies and principles are generic enough to be transferable to other educational groups. It relatively easy to imagine the MetaPark concept being applied, for example, to field trips undertaken by university students.

\section{References}

Billinghurst, M., Bowskill, J., Jessop, M. and Morphett, J. (1998), 'A wearable spatial conferencing space', 19-20 October 1998, Proceedings of the Second International Symposium on Wearable Computers, 76-83.

Billinghurst, M., Bowskill, J., Dyer, N. and Morphett, J. (1998), 'An evaluation of spatial information displays on a wearable computer', IEEE Computer Graphics and Applications, 24-31.

Buxton, W. (1997), 'Living in augmented reality: ubiquitous media and reactive environments', in Finn, K., Sellen, A. and Wilber, S. (eds.), Video Mediated Communication, Hillsdale, N.J.: Erlbaum, 363-84, http://www.dgp.utoronto.ca/OTP/papers/bill.buxton/augmentedReality.html.

Dyer, N. and Bowskill, J. (1999), 'Ubiquitous communications and media: steps towards a wearable learning tool', Proceedings of the British Computer Society Conference on Digital Media Futures, 13-15 April 1999, Bradford, UK.

Pascoe, J. (1998), 'Adding generic contextual capabilities to wearable computers', Proceedings of the Second International Symposium on Wearable Computers, 92-9.

Starner, T., Mann, S., Rhodes, B., Levine, J., Healey, J., Kirsch, D., Picard, R. and Pentland, A. (1997), 'Augmented reality through wearable computing', Presence, 6 (4).

Van de Velde, W. (1997), 'Co-habited mixed realities', Proceedings of the International Workshop on 'Community Ware', Tokyo, Japan: IJCOAI.

Walker, G. and Sheppard, P. (1997), 'Telepresence - the future of telephony', BT Technology Journal, 15, 4, 11-18.

Weisner, M. (1991), 'The computer for the twenty-first century', Scientific American, 265 (3), 94-104. 